\title{
Cuidadores de crianças com leucemia: exigências do tratamento e aprendizagem de novos comportamentos
}

\author{
Marina Kohlsdorf \\ Áderson Luiz Costa Junior \\ Universidade de Brasília
}

\begin{abstract}
Resumo
O câncer na faixa pediátrica representa um contexto de perdas, incertezas, dor física e diversas dificuldades para o paciente e seus familiares. O objetivo deste estudo foi investigar as demandas específicas vivenciadas por 30 cuidadores pediátricos, bem como comportamentos favoráveis adquiridos ao longo do semestre inicial de tratamento para leucemias, a partir de entrevistas semi-estruturadas aplicadas em dois momentos. Os resultados indicaram exigências específicas, como necessidade de maior atenção e monitoramento do paciente e aumento dos gastos financeiros. Houve relatos importantes sobre a aprendizagem de novos padrões de comportamento referidos como favoráveis, tais como aquisição de comportamentos mais adaptativos a demandas do ambiente, percepção de maior autoeficácia e atuação mais ativa no tratamento, aprendizagem sobre procedimentos médicos invasivos e destituição de crenças disfuncionais associadas ao câncer. O estudo aprofunda dados indicados na literatura e destaca a importância de mais intervenções psicossociais junto a pacientes pediátricos e cuidadores.
\end{abstract}

Palavras-chave: câncer; leucemia; aprendizagem.

\begin{abstract}
Caregivers of children with leukemia: treatment demands and learning related to new behaviors. The pediatric cancer represents a context of losses, uncertainties, physical pain and several difficulties to the patient and family. The main goal of this study was to investigate specific demands experienced by 30 pediatric caregivers, as well as favourable behaviors acquired, along the first semester of leukemia treatment, using semi-structured interviews carried out in two moments. The results indicate specific demands, such as the need of more attention and patient monitoring, and the increase of financial costs. There were relevant reports about learning new behavioral patterns, referred as beneficial, such as the acquisition of more adaptative behaviors related to environmental demands, awareness of a higher self-efficacy and a more active role in treatment, learning related to invasive medical procedures and destitution of dysfunctional beliefs related to cancer. This study corroborates data indicated in literature and highlights the importance of more psychosocial interventions concerning pediatric patients and caregivers.
\end{abstract}

Keywords: cancer; leukemia; learning.

A pesar dos crescentes avanços nos índices de sobrevida, técnicas diagnósticas, alternativas terapêuticas e campanhas de detecção precoce, o câncer na infância ainda está associado a uma representação simbólica de morte, incurabilidade, perdas, intenso sofrimento e descontrole corporal (Carvalho, 2002; Shiota, Santos, \& Miyazaki, 2004; Vrijmoet-Wiersma et al., 2008). Caracterizadas como um grupo de mais de 200 doenças que envolvem alterações no processo de divisão celular e multiplicação disfuncional de células, as neoplasias pediátricas podem ocorrer na criança ou adolescente independentemente de sua idade, sexo, cor ou etnia, condição nutricional ou socioeconômica.

O impacto adverso do câncer sobre indivíduos e grupos familiares fez surgir a psico-oncologia, na década de 50 do século XX, uma área de interface entre a psicologia e a oncologia, que utiliza conhecimentos educacionais, profissionais e científico-metodológicos para: (a) identificar o papel de aspectos psicossociais sobre a etiologia e o desenvolvimento do câncer; (b) investigar fatores psicossociais envolvidos com a prevenção e a reabilitação; e (c) incentivar a sistematização de conhecimentos que subsidiem a assistência integral ao paciente e família e a formação de profissionais de saúde qualificados (Gimenes, 1994).

Considerando a literatura em psico-oncologia pediátrica, Costa Junior (2001) e também Patenaude e Kupst (2005) destacam que contínuos avanços científicos e tecnológicos das 
ciências da saúde têm proporcionado um aumento significativo dos índices de sobrevivência ao câncer na infância e na adolescência. Por conseguinte, novos interesses de pesquisa despontam na literatura como potencialmente relevantes como, por exemplo, os efeitos em longo prazo da exposição a contextos de quimioterapia e radioterapia sobre o processo de desenvolvimento das crianças, tanto em termos orgânicos (físicos, funcionais) quanto em condições psicossociais.

O planejamento do tratamento do câncer na infância, considerando a probabilidade crescente de que a criança pode curar-se, deve incluir uma exposição aos contextos de tratamento, informação e comunicação voltados às necessidades psicossociais específicas de cada paciente e seus familiares, com a mesma importância com que se define o protocolo de quimioterapia de cada caso (Costa Junior, 2001). Embora se observe um crescente interesse pela investigação das reações comportamentais de crianças, familiares e membros da equipe de saúde frente ao diagnóstico, ao tratamento e à sobrevivência ao câncer, ainda são necessários mais trabalhos que investiguem, de modo sistematizado, as influências de variáveis psicossociais sobre o ambiente de cuidados em onco-hematologia pediátrica (Brody \& Simmons, 2007; Kars, Duijnstee, Pool, Delden, \& Grypdonck, 2008). De modo especial, é relevante investigar as principais dificuldades e desafios vivenciados pelo paciente e seus familiares, quais alterações e adaptações comportamentais são necessárias em função do curso de tratamento e ainda mudanças de comportamentos que podem ser valorizadas como benéficas, a partir do tratamento.

A criança em tratamento antineoplásico é exposta a contextos de repetidos procedimentos médicos invasivos, efeitos colaterais, interrupção da rotina escolar e social, suspensão de atividades de lazer, alteração da dieta, mudanças na autoimagem e autoconceito, sentimentos de incerteza sobre a evolução do tratamento, internações periódicas, dor, perdas relevantes que prejudicam sua socialização e interferem de modo adverso sobre a manutenção de seus relacionamentos cotidianos (Brody \& Simmons, 2007; Kars et al., 2008; McGrath, Paton \& Huff, 2005; Shiota et al., 2004).

Os pais ou responsáveis pelas práticas educativas da criança ou adolescente em tratamento são expostos a maiores responsabilidades em cuidados parentais, administrando exigências do tratamento (tais como medicação, intercorrências, efeitos colaterais, acompanhamento em consultas e exames invasivos), adaptando rotinas familiares e profissionais, manejando os comportamentos do paciente e de seus irmãos saudáveis e ainda lidando com seus próprios medos, expectativas e ameaças de recaídas (Clarke, 2004; Clarke \& Fletcher, 2003; Kars et al., 2008; Keegan-Wells et al., 2002; McGrath et al., 2005; Shiota et al., 2004).

A literatura indica diversas perturbações psicológicas vivenciadas pelos pais ou cuidadores pediátricos, em especial episódios depressivos, manifestações de ansiedade, sintomas de estresse pós-traumático e vivências de distress, além de custos financeiros elevados, mudanças importantes na rotina profissional e alterações da dinâmica familiar (Brody \& Simmons, 2007; Dolgin et al., 2007; Gerhardt et al., 2007; Kazak,
Boeving, Alderfer, Hwang \& Reilly, 2005; Pai et al., 2007; Steele, Dreyer, \& Phipps, 2004; Vrijmoet-Wiersma et al., 2008; Wijnberg-Williams, Kamps, Klip, \& Hoekstra-Weebers, 2006).

Entretanto, alguns estudos apontam que os pais ou cuidadores podem se adaptar de forma satisfatória à nova condição familiar, com perturbações psicológicas pouco diferentes daquelas vivenciadas por pais de crianças não expostas a contextos de tratamento onco-hematológico (Clarke \& Fletcher, 2004; Greening \& Stoppelbein, 2007). Apesar de relatos de preocupação dos pais com o retorno da criança às atividades curriculares e disciplinares que foram alteradas em função do tratamento, há também relatos de benefícios às crianças relacionados ao sucesso no tratamento, tais como maior independência e maturidade psicossocial (Clarke \& Fletcher, 2004; Earle, Clarke, Eiser, \& Sheppard, 2006; Kazak et al., 2005). Além disso, o tratamento do câncer pode constituir uma experiência significativa e transformadora, atuando como ponte para mudanças estruturais e contextuais da interação familiar e promovendo uma ressignificação de valores e prioridades (Carvalho, 2002; Woodgate, 2004).

O tratamento onco-hematológico pediátrico geralmente representa desafios mais críticos para as mães dos pacientes, considerando a necessidade de tomada de decisões sobre o tratamento, administração de remédios, promoção de conforto e suporte para a criança ou adolescente e irmãos saudáveis, planejamento de atividades familiares, manejo de problemas comportamentais dos filhos, atenção a episódios de febre e outras intercorrências e supervisão de responsabilidades domésticas cotidianas (Svavarsdottir, 2005). No caso específico das leucemias, o semestre inicial de tratamento é crítico, uma vez que concentra internações mais longas e terapêutica mais agressiva e pode implicar em alterações psicológicas importantes, tais como incertezas, choque ao diagnóstico e alternância entre os estados de bem-estar e os de medo, tristeza e preocupações com a evolução da patologia (McGrath, 2002). Por outro lado, também ocorrem relatos positivos das mães sobre maior fortalecimento, amadurecimento, aprendizagem e aumento do autocontrole durante o período de tratamento de um(a) filho(a) (Alencar, Lamêgo, \& Pereira, 2007).

Grande parte das pesquisas em psico-oncologia pediátrica analisa os processos vivenciados pelos cuidadores num episódio de câncer pediátrico sob um modelo explicativo de psicopatologia (Clarke \& Fletcher, 2004; Young, Dixon-Woods \& Heney, 2002; Young, Dixon-Woods, Findlay \& Heney, 2002). Considerando que muitas famílias lidam com os estressores de forma eficaz e relatam padrões comportamentais benéficos, adquiridos e/ou modificados em função do tratamento, tornam-se necessárias pesquisas que focalizem não apenas as perturbações psicológicas vivenciadas pelos cuidadores, mas principalmente os desafios, dificuldades e exigências específicas e características de cada período de tratamento, bem como as alterações favoráveis em padrões de comportamento dos pais ou cuidadores (Björk, Wiebe, \& Hallström, 2005; Kylmä \& Juvakka, 2007; Rocha-García et al., 2002).

Desta forma, o objetivo deste estudo foi investigar quais dificuldades, demandas e exigências são vivenciadas 
por cuidadores pediátricos, bem como o relato de padrões comportamentais favoráveis adquiridos ou modificados, ao longo dos primeiros seis meses de tratamento das leucemias.

\section{Método}

\section{Participantes}

Participaram deste estudo 30 cuidadores de crianças ou adolescentes com diagnóstico de leucemia: 23 mães, cinco pais, uma tia e uma avó (responsáveis diretos pela guarda da criança ou adolescente, pelo acompanhamento durante o tratamento oncohematológico e também pelos cuidados educativos parentais). A média de idade dos cuidadores foi 33,1 anos $(D P=9,4)$ e a escolaridade variou entre o segundo ano do ensino fundamental e o ensino superior completo. Entre os cuidadores, 21 eram casados ou viviam em união estável, seis eram divorciados ou separados, dois eram solteiros e um, viúvo. Os pacientes tinham idades entre nove meses e 17 anos e o paciente era filho único em apenas sete casos.

\section{Instrumentos}

Um roteiro semi-estruturado de entrevista foi elaborado para investigar os seguintes aspectos: (a) demandas e dificuldades específicas do tratamento; (b) relatos de aprendizagem pessoal a partir do tratamento.

\section{Local}

O estudo foi realizado em um hospital da rede pública de Saúde, no Sistema único de Saúde - SUS, que atende especificamente pacientes na faixa pediátrica (zero a 18 anos de idade), além de pacientes adultos fora de possibilidades terapêuticas de cura para patologias onco-hematológicas. As principais condições pediátricas atendidas incluem leucemias, linfomas, tumores sólidos, anemia falciforme, aplasia de medula e talassemia.

\section{Cuidados éticos}

O projeto de pesquisa foi submetido a um Comitê de Ética em Pesquisa (CEP), credenciado ao Comitê Nacional de Ética em Pesquisa (CONEP), do Conselho Nacional de Saúde. O projeto foi aprovado em 22 de dezembro de 2006, sob o Protocolo $142 / 2006$. Os participantes, após convite para participar do estudo e explicação prévia sobre objetivos e metodologia da pesquisa, assinaram o Termo de Consentimento Livre e Esclarecido em duas vias.

\section{Procedimento}

O procedimento de pesquisa foi organizado em duas fases. A primeira fase ocorreu cerca de dois a três meses após o início do tratamento $(M=68$ dias; $D P=15,15)$, quando os participantes foram entrevistados pela primeira vez. A segunda fase foi realizada após cinco a seis meses $(M=163$ dias; $D P$ $=16,20)$ desde o início do tratamento, quando os participantes foram solicitados a responder novamente a mesma entrevista. As entrevistas foram gravadas em áudio e o momento da primeira e segunda entrevistas variava em função do cumprimento do protocolo de quimioterapia, de modo que todas as entrevistas fossem realizadas na mesma fase de tratamento para todos os familiares.

\section{Análise dos dados}

Para análise de frequência de relatos e seus respectivos conteúdos, as gravações das entrevistas foram transcritas para um banco eletrônico de dados e organizadas em áreas temáticas e categorias funcionais, de acordo com as proposições teóricas de Bardin (1977/2004) sobre descrições analíticas de conteúdo. Tais relatos, organizados por área temática, foram submetidos a cálculo de frequência para comparação entre os dois momentos do estudo. Desta forma, este estudo pode ser caracterizado como uma análise mista, a partir da análise qualitativa de conteúdo das respostas e organização dos temas em termos quantitativos, por frequência. Todos os depoimentos e exemplos dos participantes foram considerados neste estudo, para fins de análise, a partir do reconhecimento, em cada pergunta do tema (ou temas, caso o cuidador tenha referido mais de um assunto em cada resposta) principal contemplado pelo participante, posteriormente transformado em análise de frequências temáticas.

\section{Resultados}

Os dados obtidos foram categorizados funcionalmente em duas áreas: (a) relatos dos participantes sobre exigências do tratamento (atenção à criança e maior afetividade, maior paciência e autocontrole, monitoramento do paciente, adaptações financeiras e aumento dos gastos, conhecimento da cidade para se locomover, mudança de residência) e (b) relatos dos participantes sobre aprendizagem de novos padrões de comportamento referidos como benéficos ou favoráveis (tais como amadurecimento pessoal, desenvolvimento de autocontrole, mudanças na dinâmica afetiva familiar, lidar com procedimentos médicos, entre outros).

\section{Análise de conteúdo das respostas às entrevistas na etapa inicial do estudo}

Área 1: relatos do cuidador sobre exigências do tratamento. Diversos exemplos de demandas e exigências do tratamento foram mencionados. Maior atenção e afetuosidade à criança foram destacadas por 11 participantes como exigências ("carinho que tem que ter mais ainda" e ainda "muita atenção, compreensão"). Nove cuidadores relataram necessidade de maior paciência e autocontrole ("preciso ter saúde, força, paciência, uma coisa que eu não tinha antes, hoje eu a tentando adquirir") e 11 participantes referiram o monitoramento contínuo da criança ("mais cuidado com a alimentação, com higiene" e também "o cuidado que eu tinha triplicou, tem que olhar remédio e comida na hora certa"). Oito cuidadores enfatizaram adaptações financeiras e administração de mais gastos, somados a alterações no emprego, como desafiadores ("mais gastos né. Tem que vir pra cá sempre, de carro, aí gasta muito e antes não tinha esse gasto" e ainda "mais a questão do dinheiro, essa coisa de hospital e tudo").

Dificuldades relativas à localização e locomoção pela cidade foram abordadas por quatro participantes ("começa da gente não conhecer as coisas pra andar, pra arrumar uma coisa daqui, outra. Esse povo pede 'não, vai, desce, vai fazer uma coisa, vai 
fazer outra' e a gente não sabe nem onde é") e um cuidador destacou a mudança de residência por exigência do tratamento ("tive que me mudar da casa onde eu tava porque os médicos falaram sobre esse lance de infecção, tinha que ser tudo limpo, alimentação, visitas, cachorro, eu morava numa casa que era muito empoeirada").

Área 2: relatos do cuidador sobre aprendizagem de novos padrões de comportamento. Categoria: impacto pessoal

Os participantes relataram muitos aspectos de crescimento pessoal, a partir do modo de lidar com exigências do tratamento. Nove cuidadores destacaram amadurecimento pessoal e aquisição de novos comportamentos adaptativos às demandas ("acho que eu fiquei mais madura"). Além disso, 13 participantes referiram aquisição de autocontrole, como "muita paciência. Então, isso pra mim agora tem sido melhor porque eu a mais calma, antigamente eu não tinha muita paciência" e ainda "ficar paciente, mais tolerante". O aumento da solidariedade e do fornecimento de suporte a outras pessoas foi relatado por nove cuidadores ("aprendi a ser mais humana, a entender mais o próximo") e três participantes revelaram planos para uso futuro da aprendizagem pessoal em suporte a outros pais ("quando terminar, eu creio que vai ser uma grande experiência até em termos de ajudar outras pessoas que estiverem passando por esse problema”). Um cuidador referiu a expressão de sentimentos como crescimento pessoal.

\section{Categoria: cuidados parentais e atenção à criança}

O monitoramento da criança foi descrito como oportunidade de aprendizagem por 11 cuidadores ("não tinha horário certo, agora tem horário. É uma coisa que eu aprendi mesmo que é bom pra gente, horário"). Maior interação e mudanças na dinâmica afetiva com a criança foram destacadas por cinco participantes ("tento melhorar minha convivência com ele, ficar mais tempo com ele, dar mais carinho, brincar mais, procuro melhorar meu modo de conversar") e dois cuidadores relataram planejamentos para o futuro ("eu já estou fazendo planos, dezembro ele vai ficar melhor e eu vou viajar").

A importância de aprender a delegar o cuidado dos filhos a outras pessoas foi destacada por uma mãe e quatro participantes referiram preocupações futuras decorrentes da doença, a partir do acesso a informações sobre o tratamento ("quando ele tiver indo pra escola, a gente preocupa, não poder estar junto com pessoas que estiverem gripadas, doentes").

\section{Categoria: aspectos relacionados à leucemia}

Experiências relacionadas especificamente à leucemia incluíram uma resposta sobre aprendizagem incoerente em relação à doença e 14 relatos sobre aprendizagem coerente relacionada à enfermidade, conforme exemplificado: "a internação passada fez o exame da sorologia do Metrotexate, né? E, além da dosagem a sendo o ideal, ela conseguiu a eliminando tudo, até nisso eu já estou inteirado de saber, tem que eliminar o mais rápido possível, porque ela tem um efeito negativo no organismo depois de ter feito o efeito dela".

Melhores condições para enfrentar os procedimentos médicos e a permanência no hospital foram mencionadas por
13 participantes, conforme relatos: "eu tinha pavor de hospital, ainda hoje tenho, tive que me adaptar, fazendo tipos de exame com ele, como a punção, por exemplo, ajudar a pegar a veia, são coisas que se eu visse há um ano, dois anos atrás, era eu desmaiado. E hoje não, pra mim se tornou uma coisa natural". A destituição de crenças sobre a leucemia foi um tema abordado por 11 participantes: "aprendi que câncer também tem cura" e também "você acha que é só com o vizinho, daí de repente, acontece na sua casa". Três cuidadores ressaltaram a percepção da própria autoeficácia e/ou atuação mais ativa em relação ao tratamento do filho ("eu não ia em médico sozinha, não sabia resolver nada sozinha, ir pra hospital, porque eu era muito tímida, hoje não, hoje tiro minhas dúvidas, não tenho mais timidez a esse respeito").

\section{Análise de conteúdo das respostas às entrevistas na segunda etapa do estudo}

Área 1: relatos do cuidador sobre exigências do tratamento. Em relação a exigências do tratamento, foram relatados quatro exemplos de necessidade de maior atenção e/ou afetividade para a criança ou adolescente ("afeição, carinho, um amor com ela. Exigiu isso"). Onze participantes mencionaram maior paciência e autocontrole ("controle emocional" e ainda "muita força, muita paciência e sempre buscando a compreensão") e 11 cuidadores destacaram maior monitoramento do paciente ("exige o cuidado com o que ela come, o que ela faz porque pode ocorrer sangramento").

Adaptações financeiras e necessidades de maiores gastos foram referidos em seis relatos ("é uma falta de dinheiro da grande! Porque a gente pára de trabalhar, fica sem dinheiro. A maior dificuldade que eu a tendo agora é dinheiro" e também "o que mudou realmente é o gasto com transporte. Porque a carteirinha dela ainda não está pronta, toda vez que eu vou lá, sempre falta alguma coisa. Esperando também resolver o lance do dinheirinho que ela tem que receber, que vai ajudar muito, mas até agora nada"). Três cuidadores destacaram a necessidade de conhecer a cidade para se locomover ("essa semana eu vi que não sabia pegar ônibus antes e agora eu aprendi”).

Área 2: relatos do cuidador sobre aprendizagem de novos padrões de comportamento. Categoria: impacto pessoal

Relatos sobre o que o participante aprendeu ao longo do tratamento envolveram diversas áreas. Quase metade da amostra (14 indivíduos) ressaltou o amadurecimento pessoal, aquisição de novos padrões de comportamentos adaptativos a demandas ("veio esse problema pra me amadurecer um pouco, meus pensamentos mudaram"). O autocontrole foi referido por 12 cuidadores ("exigiu muita paciência de mim") e oito participantes ressaltaram a solidariedade e o fornecimento de suporte a outras pessoas ("você vê que não é só você que tem esse problema, tem gente com problemas iguais ou até piores, você acaba se tornando uma pessoa mais humana" ou ainda "mudou que eu passei mais a olhar as pessoas, querer ajudar, compreender, que antes eu não fazia muito"). O uso futuro da aprendizagem em suporte a outros pais foi referido por dois cuidadores ("uma mãe que recebeu o resultado hoje, eu vou saber falar pra ela pra ela não passar pelo 
que eu passei. Eu vou saber falar 'calma que não é assim"”).

$$
\text { Categoria: em relação a cuidados parentais e atenção à }
$$
criança

Descrições de aprendizagens relativas ao maior monitoramento da criança identificaram nove relatos ("passei a cuidar mais, porque meus filhos nunca tinham ficado doente, vi até que ponto você pode chegar") e 12 cuidadores destacaram maior interação social e mudanças na dinâmica afetiva com a criança ("eu rio, eu brinco, eu aprendi a ser mãe, a doença me ajudou a ser mais mãe ainda, até com os outros filhos meus, eu era mais nervosa, agora não, eu converso mais com eles, coisa que eu não fazia"). A adaptação em relação a planejamentos elaborados anteriormente foi referida por seis participantes ("eu ia fazer enfermagem, eu tive que parar para acompanhar o tratamento da minha filha, muitas coisas que eu deixei para trás") e três cuidadores destacaram preocupações futuras decorrentes da aprendizagem de informações sobre a doença ("aprendi sim muita coisa depois que cheguei nesse hospital, vi muitas coisas, hoje me sinto mais amedrontada com as coisas que eu vejo. Pessoas com o mesmo problema do seu filho, que muitas vezes não resistem, eu estou sempre assim, meio insegura, receosa").

\section{Categoria: aspectos relacionados à leucemia}

Foram categorizados 12 relatos de aprendizagem em relação à doença, coerentes com o tratamento ("quando o sangue a baixo, eu já sei como é, sempre que entregam os resultados eu olho, antes eu nem sabia o que era isso") e a adaptação aos procedimentos médicos e à permanência no hospital foi mencionada por 11 cuidadores ("tive que aprender a mexer nas máquinas do hospital, com soro"). Entre os participantes, 12 referiram a destituição de crenças disfuncionais relativas à doença ("eu não esperava que existia essa doença, pra mim não existia no Brasil", "no início a gente fica meio apavorado, porque antigamente era um tipo de doença que a pessoa já se condenava, a fadado a fatalidade"). Cinco cuidadores relataram a percepção de autoeficácia e/ou atuação mais ativa ao longo do tratamento ("aprende que, mesmo que você ache que não tem nada com seu filho ou com você mesmo, é bom você sempre a fazendo um exame, uma prevenção, né? Acho que o aprendizado é total, geral"). A Tabela 01 mostra as frequências de relatos obtidos em cada área, nas duas etapas de entrevista.

Tabela 1

Frequências de relatos nas fases de entrevistas

\begin{tabular}{|c|c|c|}
\hline Frequências de relatos nas entrevistas 1 e 2 , por área & Entr.1 & Entr.2 \\
\hline Área 1: relatos do cuidador sobre exigências do tratamento & $(f)$ & (f) \\
\hline a) Atenção à criança e maior afetividade & 11 & 4 \\
\hline b) Maior paciência e autocontrole & 9 & 11 \\
\hline c) Monitoramento do paciente & 11 & 11 \\
\hline d) Adaptações financeiras e aumento dos gastos & 8 & 6 \\
\hline e) Conhecer a cidade para se locomover & 4 & 3 \\
\hline f) Mudança de residência & 1 & 0 \\
\hline \multicolumn{3}{|l|}{$\begin{array}{l}\text { Área 2: relatos do cuidador sobre aprendizagem de novos padrões de comportamento } \\
\text { Categoria: impacto pessoal }\end{array}$} \\
\hline a) Amadurecimento pessoal & 9 & 14 \\
\hline b) Desenvolvimento de autocontrole & 13 & 12 \\
\hline c) Fornecimento de suporte a outras pessoas, solidariedade & 9 & 8 \\
\hline d) Uso futuro da aprendizagem pessoal como fonte de suporte a outros pais & 3 & 2 \\
\hline e) Expressão de sentimentos, protestar & 1 & 0 \\
\hline \multicolumn{3}{|l|}{ Categoria: em relação a cuidados parentais e atenção à criança } \\
\hline a) Monitoramento da criança & 11 & 9 \\
\hline b) Maior interação e mudança na dinâmica afetiva com a criança & 5 & 12 \\
\hline c) Modificação de planejamentos & 2 & 6 \\
\hline d) Preocupações futuras em decorrência da doença & 4 & 3 \\
\hline e) Delegar cuidados de irmãos saudáveis a outras pessoas & 1 & 0 \\
\hline \multicolumn{3}{|l|}{ Categoria: aspectos relacionados à leucemia } \\
\hline a) Aprendizagem em relação à doença & 15 & 12 \\
\hline b) Lidar com procedimentos médicos e permanência em hospital & 13 & 11 \\
\hline c) Destituição de crença & 11 & 12 \\
\hline d) Percepção de autoeficácia do cuidador e/ou atuação mais ativa no tratamento & 3 & 5 \\
\hline
\end{tabular}

\section{Discussão}

Os resultados confirmam muitos dados apontados pela literatura, que destaca diversas exigências impostas aos cuidadores durante um tratamento onco-hematológico pediátrico (Brody \& Simmons, 2007; Clarke, 2004; Clarke \& Fletcher, 2003; Kars et al., 2008; Keegan-Wells et al., 2002; McGrath et al., 2005; Shiota et al., 2004). Especificamente, as demandas incluem maior atenção e afetividade dirigidas ao paciente, desenvolvimento de autocontrole, maior monitoramento do paciente, aumento dos gastos, conhecimento da cidade para propiciar locomoção e mudanças de residência, em função de exigências do tratamento. Tais demandas podem contribuir para aumentar custos comportamentais consequentes ao tratamento, possibilitando contextos propícios para o surgimento de perturbações psicológicas (Dolgin et al., 2007; Gerhardt et al., 2007; Kazak et al., 2005; McGrath et al., 2005; Pai et al., 2007; Steele et al., 2004; Vrijmoet-Wiersma et al., 2008; WijnbergWilliams et al., 2006).

Além de corroborar dados indicados na literatura, o presente estudo aprofunda a análise sobre quais elementos estão especificamente relacionados às dificuldades impostas pelo tratamento, trazendo duas contribuições importantes à área: (a) o efetivo detalhamento sobre a natureza e qualidade das dificuldades enfrentadas; e (b) o acompanhamento processual das mudanças vivenciadas ao longo do tempo de tratamento. A partir dos resultados apresentados, torna-se possível compreender, de modo mais específico, quais são as exigências características 
em cada fase de tratamento, promovendo subsídios importantes para intervenções psicossociais mais eficientes e focalizadas em demandas específicas. A comparação entre as duas etapas distintas permite verificar o processo e as mudanças específicas vivenciadas pelos cuidadores, ao longo do semestre inicial de tratamento, bem como suas principais dificuldades em cada momento, conforme destacado a seguir.

Destaca-se a relativa diminuição de relatos sobre exigências e demandas entre as duas fases da pesquisa, indicador de que os primeiros meses de tratamento para leucemias são caracterizados por maiores exigências para adaptação ao contexto de tratamento. Ao longo do semestre inicial, os cuidadores começam a lidar melhor com tais demandas e dificuldades, resultando possivelmente na diminuição de relatos sobre dificuldades enfrentadas. Além disso, o protocolo de tratamento para leucemias é caracterizado por dois a três meses iniciais de terapia quimioterápica mais agressiva e internações mais frequentes e prolongadas, com gradual diminuição da frequência e duração das internações e terapêutica menos aversiva, fator que também pode influenciar a diminuição na frequência de relatos sobre exigências e demandas do tratamento, ao longo da pesquisa. Destaca-se a necessidade de intervenções mais específicas e que possam ser estendidas ao longo do semestre inicial de tratamento, e não apenas durante a fase de diagnóstico. Este investimento em intervenções de acompanhamento pode promover uma melhor adaptação de cuidadores às demandas e prepara-los melhor para a retomada de atividades pessoais, profissionais e familiares, após término do tratamento.

Os resultados mostram ainda relatos importantes sobre alterações na rotina profissional dos cuidadores, que acarretam a necessidade de adaptações financeiras e aumento dos gastos, dados também indicados na literatura (Björk et al., 2005; McGrath et al., 2005; Rocha-García et al., 2002). Ressalta-se que ainda são escassos, na literatura brasileira, estudos que focalizem o impacto do tratamento pediátrico na condição financeira da família, bem como a importância de implementar Políticas Públicas específicas e as questões de cunho burocrático/ administrativo envolvidas nessa condição. A demissão ou afastamento do emprego, somados à diminuição no horário de trabalho e ausência do benefício financeiro, garantido em lei para pacientes em tratamento onco-hematológico, constituem fatores críticos que prejudicam imensamente a qualidade de vida do acompanhante e do paciente. $\mathrm{O}$ apoio governamental, no sentido de promover condições instrumentais de subsistência, locomoção e moradia, torna-se imprescindível, sem considerar que são direitos fundamentais do cidadão. $\mathrm{O}$ fornecimento deste apoio instrumental por parte do governo torna-se prioritário, a partir do cumprimento e garantia dos benefícios previstos em lei para pacientes e acompanhantes durante tratamento. As alterações na rotina profissional dos cuidadores constituem uma dificuldade adicional ao tratamento que poderia ser imediatamente solucionada ou minimizada a partir da eficiência na concessão de benefícios, bolsas e passes-livre para deslocamento.

Um aspecto importante ressaltado neste estudo refere-se ao intenso número de relatos indicativos de aprendizagem e/ou modificação de padrões de comportamento por parte dos cuidadores, estimados como benéficos ou favoráveis, conforme também indicado em outros estudos (Alencar et al., 2007; Clarke \& Fletcher, 2004; Earle et al., 2006; Kazak et al., 2005). A literatura indica uma tendência a compreender o impacto do tratamento sob uma perspectiva psicopatológica (Clarke \& Fletcher, 2004; Young, Dixon-Woods \& Heney, 2002; Young, Dixon-Woods, Findlay \& Heney, 2002), limitando as possibilidades de investigação e intervenção na área. $\mathrm{O}$ presente estudo destaca a importância de considerar aspectos de aprendizagem benéfica referida pelos cuidadores, oferecendo subsídios importantes para outras pesquisas subsequentes.

Nas duas etapas de entrevistas, todos os participantes referiram, pelo menos, um aspecto relativo ao amadurecimento e crescimento pessoais, exemplificados como aquisição de comportamentos mais adaptativos a demandas do ambiente e maior autocontrole, solidariedade e aumento do suporte fornecido a outros pais, percepção de autoeficácia e atuação mais ativa no tratamento. Cabe destacar também relatos de aprendizagem referentes a melhorias na interação afetiva familiar e nas práticas educativas parentais, que aumentaram em frequência na última etapa e se caracterizaram especialmente por mudanças na dinâmica e interação afetivas com a criança ou adolescente. Esses resultados indicam que o tratamento do câncer pode constituir uma experiência transformadora, proporcionando mudanças estruturais e contextuais da interação familiar e ainda promovendo uma ressignificação de valores e prioridades de vida (Carvalho, 2002; Woodgate, 2004).

Em relação à aprendizagem sobre procedimentos médicos invasivos, diagnóstico, tratamento e permanência em hospitais, também ocorreram relatos importantes nas etapas do estudo. Destaca-se a presença, na última fase, de descrições sobre preocupações futuras com complicações e intercorrências, possivelmente em decorrência da aquisição de informações sobre a doença e do contato com outros casos semelhantes à condição do paciente. Torna-se também relevante ressaltar a presença significativa de relatos sobre destituição de crenças disfuncionais associadas ao câncer de modo geral, dado que pode indicar a necessidade de difundir, entre a população brasileira, conhecimentos atualizados sobre importância do acompanhamento pediátrico, detecção precoce, tipos de tratamento e chances de sucesso.

Concluindo, o estudo apresenta resultados que corroboram dados indicados na literatura, mas aprofunda a análise sobre as dificuldades referidas pelos cuidadores, ao permitir uma análise mais minuciosa sobre o processo e as mudanças vivenciadas nos primeiros meses de tratamento. Os resultados permitem destacar as exigências e demandas específicas enfrentadas por cuidadores pediátricos durante um tratamento oncohematológico e promovem o detalhamento mais específico sobre a natureza e qualidade dessas dificuldades, indicando padrões de comportamento que podem ser modificados de forma benéfica ou favorável ao longo deste período.

Considerando as dificuldades e demandas intensas inerentes a um tratamento, destaca-se a necessidade de mais pesquisas que investiguem, de modo sistematizado e em caráter longitudinal, a vivência de cuidadores, pacientes pediátricos, outros familiares e ainda dos profissionais de saúde envolvidos, ao longo de um tratamento. Além disso, ressalta-se a relevância de intervenções 
psicossociais junto a pacientes e seus respectivos cuidadores, a fim de contribuir para diminuir custos comportamentais associados ao tratamento e proporcionar uma melhor qualidade de vida ao paciente e seus familiares, durante o contexto potencialmente aversivo de tratamento onco-hematológico.

\section{Referências}

Alencar, M. P., Lamêgo, A. N., \& Pereira, L. M. C. (2007). Familias com crianças com câncer. Recuperado de http://www.cientefico.frb.br

Bardin, L. (2004). Análise de conteúdo. (L. A. Reto e A. Pinheiro, Trad.). Lisboa: Edições 70 LDA. (Trabalho original publicado em 1977)

Björk, M., Wiebe, T., \& Hallström, I. (2005). Striving to survive: families' lived experiences when a child is diagnosed with cancer. Journal of Pediatric Oncology Nursing, 22(5), 265-275.

Brody, A. C., \& Simmons, L. A. (2007). Family resiliency during childhood cancer: the father's perspective. Journal of Pediatric Oncology Nursing, 24(3), 152-165.

Carvalho, M. M. (2002). Psico-oncologia: história, características e desafios. Psicologia USP, 13(1), 151-166.

Clarke, J. (2004). Whose power/authority/knowledge? Conundrums in the experiences of parents whose children have cancer. Social Work in Health Care, 40(2), 13-35.

Clarke, J. N., \& Fletcher, P. (2003). Communication issues faced by parents who have a child diagnosed with cancer. Journal of Pediatric Oncology Nursing, 20(4), 175-191.

Clarke, J. N., \& Fletcher, P. (2004). Parents as advocates: stories of surplus suffering when a child is diagnosed and treated for cancer. Social Work on Health Care, 39(2), 102-127.

Costa Junior, A. L. (2001). O desenvolvimento da psico-oncologia: implicações para a pesquisa e intervenção profissional em saúde. Psicologia: Ciência e Profissão, 21(2), 36-43.

Dolgin, M. J., Phipps, S., Fairclough, D. L., Sahler, O. J., Askins, M., Noll, R. B., ... Katz, E. R. (2007). Trajectories of adjustment in mothers of children with newly diagnosed cancer: A natural history investigation. Journal of Pediatric Psychology, 32(7), 771-782.

Earle, E. A., Clarke, S. A., Eiser, C., \& Sheppard, L. (2006). Building a new normality: Mothers' experiences of caring for a child with acute lymphoblastic leukaemia. Child: Care, Health and Development, 33(2), 155-160.

Gerhardt, C. A., Gutzwiller, J., Huiet, K. A., Fischer, S., Noll, R. B., \& Vannatta, K. (2007). Parental adjustment to childhood cancer: A replication study. Families, Systems \& Health, 25(3), 263-275.

Gimenes, M. G. G. (1994). Definição, foco de estudo e intervenção. In M. M. M. J. Carvalho (Org.), Introdução à Psiconcologia (pp. 35-56). São Paulo: Editorial Psy.

Greening, L., \& Stoppelbein, L. (2007). Brief report: pediatric cancer, parental coping style, and risk for depressive, posttraumatic stress, and anxiety symptoms. Journal of Pediatric Psychology, 32(10), 1272-1277.

Kars, M. C., Duijnstee, M. S. H., Pool, A., Delden, J. J. M., \& Grypdonck, M. H. F. (2008). Being there: parenting the child with acute lymphoblastic leukaemia. Journal of Clinical Nursing, 17(12), 1553-1562.
Kazak, A. E., Boeving, C. A., Alderfer, M. A., Hwang, W. T., \& Reilly, A. (2005) Posttraumatic stress symptoms during treatment in parents of children with cancer. Journal of Clinical Oncology, 23(30), 7406-7410.

Keegan-Wells, D., James, K., Stewart, J. L., Moore, I. M., Kelly, K. P., Moore, B., Bond, D., ... Speckhart, B. (2002). The care of my child with cancer: a new instrument to measure caregiving demand in parents of children with cancer. Journal of Pediatric Nursing, 17(3), 201-210.

Kylmä, J., \& Juvakka, T. (2007). Hope in parents of adolescents with cancer : factors endangering and engendering parental hope. European Journal of Oncology Nursing, 11(3), 262-271.

McGrath, P. (2002). Beginning treatment for childhood acute lymphoblastic leukemia: insights from the parents' perspective. Oncology Nursing Forum, 29(6), 988-996.

McGrath, P., Paton, M. A., \& Huff, N. (2005). Beginning treatment for pediatric acute myeloid leukemia: the family connection. Issues in Comprehensive Pediatric Nursing, 28(2), 97-114.

Pai, A. L. H., Greenley, R. N., Lewandowski, A., Drotar, D., Youngstrom, E., \& Peterson, C. C. (2007). A meta-analytic review of the influence of pediatric cancer on parent and family functioning. Journal of Family Psychology, 21(3), 407-415.

Patenaude, A. F., \& Kupst, M. J. (2005). Psychosocial functioning in pediatric cancer. Journal of Pediatric Psychology, 30(1), 9-27.

Rocha-García, A., Del Río, A. A., Hérnandez-Peña, P., Martínez-García, M. C., Marín-Palomares, T., \& Lazcano-Ponce, E. (2002). The emotional response of families to children with leukemia at the lower socio-economic level in central Mexico: a preliminary report. Psycho-oncology, 12(1), 78-90.

Shiota, C. M., Santos, A. R. R. \& Miyazaki, M. C. O. S. (2004). Problemas de comportamento em crianças com câncer: o papel dos pais. In M. Z. S. Brandão, F. C. S. Conte, F. S. Brandão, Y. K. Ingberman, C. B. Moura, V. M. Silva, \& S. M. Oliane (Orgs.), Sobre comportamento e cognição: estendendo a psicologia comportamental e cognitiva aos contextos da saúde, das organizações, das relações pais e filhos e das escolas (Vol. 14, pp. 261-266). Santo André: ESETEC.

Steele, R. G., Dreyer, M. L., \& Phipps, S. (2004). Patterns of maternal distress among children with cancer and their association with child emotional and somatic distress. Journal of Pediatric Psychology, 29(7), 507-517.

Svavarsdottir, E. K. (2005). Gender and emotions: icelandic parents experiencing childhood cancer. International Journal of Nursing Studies, 42(5), 531-538.

Vrijmoet-Wiersma, C. M. J., Van Klink, J. M. M., Kolk, A. M., Koopman, H. M., Ball, L. M., \& Egeler, R. M. (2008). Assessment of parental psychological stress in pediatric cancer: a review. Journal of Pediatric Psychology, 33(1), $1-13$.

Wijnberg-Williams, B. J., Kamps, W. A., Klip, E. C., \& Hoekstra-Weebers, J. E. H. M. (2006). Psychological distress and the impact of social support on fathers and mothers of pediatric cancer patients: Long-term prospective results. Journal of Pediatric Psychology, 31(8), 785-792.

Woodgate, R. L. (2004). Cancer symptom transition periods of children and families. Journal of Advanced Nursing, 46(4), 358-368.

Young, B., Dixon-Woods, M., \& Heney, D. (2002). Identity and role in parenting a child with cancer. Pediatric Rehabilitation, 5(4), 209-214.

Young, B., Dixon-Woods, M., Findlay, M., \& Heney, D. (2002). Parenting in a crisis: conceptualizing mothers of children with cancer. Social Science \& Medicine, 55(10), 1835-1847. 
234 M. Kohlsdorf \& A. L. Costa Junior

Marina Kohlsdorf, mestre em Processos de Desenvolvimento Humano e Saúde pela Universidade de Brasília, é doutoranda em Processos de Desenvolvimento Humano e Saúde na mesma universidade. Endereço para correspondência: SQS 103, Bloco B, ap. 101. CEP: 70342-020. Brasília - DF. Telefones: (61)3224-4452/ (61)8172-9030. E-mail:marinak@unb.br

Áderson Luiz Costa Junior, doutor em Psicologia pela Universidade de Brasília, pós- doutor em Psicologia pela Universidade Estadual de Campinas, é professor Adjunto na Universidade de Brasília. E-mail: aderson@unb.br 